PROCEEDINGS OF THE

AMERICAN MATHEMATICAL SOCIETY

Volume 130, Number 7 , Pages 1905-1912

S 0002-9939(01)06269-4

Article electronically published on December 27, 2001

\title{
THE F-DEPTH OF AN IDEAL ON A MODULE
}

\author{
RENCAI LÜ AND ZHONGMING TANG
}

(Communicated by Wolmer V. Vasconcelos)

\begin{abstract}
Let $I$ be an ideal of a Noetherian local ring $R$ and $M$ a finitely generated $R$-module. The $\mathrm{f}$-depth of $I$ on $M$ is the least integer $r$ such that the local cohomology module $H_{I}^{r}(M)$ is not Artinian. This paper presents some part of the theory of f-depth including characterizations of f-depth and a relation between $\mathrm{f}$-depth and f-modules.
\end{abstract}

\section{INTRODUCTION}

Let $(R, \mathfrak{m})$ be a (commutative) Noetherian local ring, $I$ a proper ideal of $R$ and $M$ a finitely generated $R$-module. It is well known that the depth, $\operatorname{depth}(I, M)$, of $I$ on $M$, i.e., the length of a maximal $M$-regular sequence in $I$, is the least integer $r$ such that the local cohomology module $H_{I}^{r}(M) \neq 0$. Faltings [2] proved that the least integer $r$ such that $H_{I}^{r}(M)$ is not finitely generated is

$$
\min \left\{\operatorname{depth}\left(M_{\mathfrak{p}}\right)+\operatorname{ht}((I+\mathfrak{p}) / \mathfrak{p}) \mid \mathfrak{p} \nsupseteq I\right\} .
$$

Now we consider the problem of what is the least integer $r$ such that $H_{I}^{r}(M)$ is not Artinian. In [4, Melkersson showed that, when $\operatorname{Supp}(M / I M) \nsubseteq\{\mathfrak{m}\}$, the least integer $r$ such that $H_{I}^{r}(M)$ is not Artinian is

$$
\min \left\{\operatorname{depth}\left(I R_{\mathfrak{p}}, M_{\mathfrak{p}}\right) \mid \mathfrak{p} \in \operatorname{Supp}(M / I M) \backslash\{\mathfrak{m}\}\right\},
$$

and called this integer the f-depth (filter depth) of $I$ on $M$. In this paper, for any proper ideal $I$, we define the f-depth of $I$ on $M$ as the length of a maximal $M$-filter regular sequence in $I$. Then it turns out that our f-depth of $I$ on $M$ is the least integer $r$ such that $H_{I}^{r}(M)$ is not Artinian for any proper ideal $I$ and, in the case $\operatorname{Supp}(M / I M) \nsubseteq\{\mathfrak{m}\}$, our f-depth coincides with the one of Melkersson.

After summarizing some results about filter regular sequences in section 2, we define f-depth in section 3. Then, we characterize f-depth by Ext, Koszul complexes and local cohomology modules. Section 4 contains an equivalent condition using $\mathrm{f}$-depth for an $R$-module to be an f-module which is similar to a Cohen-Macaulay module.

Received by the editors July 26, 2000 and, in revised form, January 16, 2001.

2000 Mathematics Subject Classification. Primary 13C15, 13D45, $14 \mathrm{~B} 15$.

Key words and phrases. f-depth, f-modules, local cohomology modules.

This work was supported by the National Natural Science Foundation of China.

(C)2001 American Mathematical Society 


\section{Preliminaries on Filter Regular SEQUenCes}

Throughout the paper, let $(R, \mathfrak{m})$ be a (commutative) Noetherian local ring and $M$ a finitely generated $R$-module. For any submodule $N$ of $M$, we use $N:_{M}\langle\mathfrak{m}\rangle$ to denote the submodule $\left\{m \in M \mid \mathfrak{m}^{n} m \subseteq N\right.$ for some $\left.n>0\right\}$.

Definition 2.1. Let $x_{1}, \ldots, x_{n} \in \mathfrak{m}$. If, for $i=1, \ldots, n$,

$$
\left(x_{1}, \ldots, x_{i-1}\right) M:_{M} x_{i} \subseteq\left(x_{1}, \ldots, x_{i-1}\right) M:_{M}\langle\mathfrak{m}\rangle
$$

then we say that $x_{1}, \ldots, x_{n}$ is an $M$-filter regular sequence.

Notice that $x \in \mathfrak{m}$ is $M$-filter regular if and only if $x \notin \bigcup_{\mathfrak{p} \in \operatorname{Ass}_{R}(M) \backslash\{\mathfrak{m}\}} \mathfrak{p}$ and $x_{1}, x_{2}, \ldots, x_{n}$ is an $M$-filter regular sequence if and only if $x_{1}$ is $M$-filter regular and $x_{2}, \ldots, x_{n}$ is an $M / x_{1} M$-filter regular sequence. For a finitely generated $R$ module $N$, the length of $N$ is denoted by $\ell(N)$. Then $\ell(N)<\infty$ if and only if $\operatorname{dim}(N) \leq 0$. Thus $x_{1}, \ldots, x_{n}$ is an $M$-filter regular sequence is equivalent to

$$
\ell\left(\left(x_{1}, \ldots, x_{i-1}\right) M:_{M} x_{i} /\left(x_{1}, \ldots, x_{i-1} M\right)\right)<\infty, i=1, \ldots, n .
$$

On the other hand, we are reminded that $y_{1}, \ldots, y_{s} \in R$ is a poor $M$-regular sequence if

$$
\left(y_{1}, \ldots, y_{i-1}\right) M:_{M} y_{i}=\left(y_{1}, \ldots, y_{i-1}\right) M, i=1, \ldots, s,
$$

and, if furthermore $\left(y_{1}, \ldots, y_{s}\right) M \neq M$, we call $y_{1}, \ldots, y_{s}$ an $M$-regular sequence. Then $x_{1}, \ldots, x_{n}$ is an $M$-filter regular sequence if and only if, for any $\mathfrak{p} \in \operatorname{Supp}(M) \backslash$ $\{\mathfrak{m}\}, x_{1} / 1, \ldots, x_{n} / 1$ is a poor $M_{\mathfrak{p}}$-regular sequence, and, if $x_{1}, \ldots, x_{n}$ is an $M$ filter regular sequence, then $x_{1} / 1, \ldots, x_{n} / 1$ is an $M_{\mathfrak{p}}$-regular sequence for any $\mathfrak{p} \in$ $\operatorname{Supp}(M) \backslash\{\mathfrak{m}\}$ with $x_{1}, \ldots, x_{n} \in \mathfrak{p}$.

Since, for any poor $M$-regular sequence $y_{1}, \ldots, y_{s}$ and any integers $i_{j}>0, j=$ $1, \ldots, s, y_{1}^{i_{1}}, \ldots, y_{s}^{i_{s}}$ is also a poor $M$-regular sequence, it follows that if $x_{1}, \ldots, x_{n}$ is an $M$-filter regular sequence, then, for any integers $i_{j}>0, j=1, \ldots, n, x_{1}^{i_{1}}, \ldots$, $x_{n}^{i_{n}}$ is also an $M$-filter regular sequence. However, a permutation of a filter regular sequence is not necessarily filter regular again. For example, let $K\left[x_{1}, x_{2}\right]$ be a polynomial ring and $K$ a field. Set $R=K\left[x_{1}, x_{2}\right]_{\left(x_{1}, x_{2}\right)}$ and $M=R \oplus R /\left(x_{2}^{2}\right)$. Then, as $0:_{M} x_{1}=0$ and $0:_{M / x_{1} M} x_{2} \subseteq R /\left(x_{1}, x_{2}^{2}\right)$ has finite length, it follows that $x_{1}, x_{2}$ is an $M$-filter regular sequence. But, as the prime ideal $\left(x_{2}\right) \in \operatorname{Ass}_{R}(M) \backslash\left\{\left(x_{1}, x_{2}\right)\right\}$ and $x_{2} \in\left(x_{2}\right)$, we see that $x_{2}$ is not $M$-filter regular, hence, $x_{2}, x_{1}$ is not an $M$-filter regular sequence.

For any $x_{1}, \ldots, x_{n} \in R$, let $H_{i}\left(x_{1}, \ldots, x_{n} ; M\right)$ be the $i$-th homology module of the Koszul complex $K .\left(x_{1}, \ldots, x_{n} ; M\right)$ of $M$ with respect to $x_{1}, \ldots, x_{n}$. Then, we have the following:

Proposition 2.2. If $x_{1}, \ldots, x_{n}$ is an $M$-filter regular sequence, then

$$
\ell\left(H_{i}\left(x_{1}, \ldots, x_{n} ; M\right)\right)<\infty, \text { for any } i>0 \text {. }
$$

Proof. By induction on $n$. If $n=1$, then $H_{1}\left(x_{1} ; M\right)=0:_{M} x_{1}$ has finite length by definition. Now, assume that $n>1$. From the long exact sequence

$$
\begin{gathered}
\cdots \rightarrow H_{i}\left(x_{1}, \ldots, x_{n-1} ; M\right) \rightarrow H_{i}\left(x_{1}, \ldots, x_{n} ; M\right) \rightarrow H_{i-1}\left(x_{1}, \ldots, x_{n-1} ; M\right) \\
\stackrel{(-1)^{i-1} x_{n}}{\rightarrow} H_{i-1}\left(x_{1}, \ldots, x_{n-1} ; M\right) \rightarrow \cdots \rightarrow H_{0}\left(x_{1}, \ldots, x_{n} ; M\right) \rightarrow 0,
\end{gathered}
$$


we see that $\ell\left(H_{i}\left(x_{1}, \ldots, x_{n} ; M\right)\right)<\infty$ for all $i>1$ from the induction assumption that $\ell\left(H_{i}\left(x_{1}, \ldots, x_{n-1} ; M\right)\right)<\infty$ and $\ell\left(H_{i-1}\left(x_{1}, \ldots, x_{n-1} ; M\right)\right)<\infty$. For the case $i=1$, we have an exact sequence

$$
\begin{gathered}
H_{1}\left(x_{1}, \ldots, x_{n-1} ; M\right) \rightarrow H_{1}\left(x_{1}, \ldots, x_{n} ; M\right) \rightarrow H_{0}\left(x_{1}, \ldots, x_{n-1} ; M\right) \\
=M /\left(x_{1}, \ldots, x_{n-1}\right) M \stackrel{x_{n}}{\rightarrow} M /\left(x_{1}, \ldots, x_{n-1}\right) M .
\end{gathered}
$$

As $H_{1}\left(x_{1}, \ldots, x_{n-1} ; M\right)$ and $0:_{M /\left(x_{1}, \ldots, x_{n-1}\right) M} x_{n}$ have finite length, we see that $\ell\left(H_{1}\left(x_{1}, \ldots, x_{n} ; M\right)\right)<\infty$. Hence, for all $i>0, \ell\left(H_{i}\left(x_{1}, \ldots, x_{n} ; M\right)\right)<\infty$.

Notice that the converse of Proposition 2.2 is not true because the conditions $\ell\left(H_{i}\left(x_{1}, \ldots, x_{n} ; M\right)\right)<\infty$ for all $i>0$ do not depend on the order of $x_{1}, \ldots, x_{n}$, but the filter regularity does.

\section{Characterizations of F-DePth}

In order to show that f-depth is well-defined, we need the following:

Lemma 3.1. Let $I \subseteq \mathfrak{m}$ be an ideal. If $\ell\left(\operatorname{Hom}_{R}(R / I, M)\right)<\infty$, then there exists $x \in I$ which is $M$-filter regular.

Proof. Assume the contrary. Then $I \subseteq \bigcup_{\mathfrak{p} \in \operatorname{Ass}_{R}(M) \backslash\{\mathfrak{m}\}} \mathfrak{p}$, so that $I \subseteq \mathfrak{p}$ for some $\mathfrak{p} \in \operatorname{Ass}_{R}(M) \backslash\{\mathfrak{m}\}$. Hence $\mathfrak{p}=\operatorname{ann}_{R}(m)$ for some $m \in M$, so $\operatorname{dim}(R m)>0$. But $I m=0$, so $R m \subseteq 0:_{M} I \cong \operatorname{Hom}_{R}(R / I, M)$. Thus $\operatorname{dim}\left(\operatorname{Hom}_{R}(R / I, M)\right)>0$, a contradiction.

Proposition 3.2. Let $I \subseteq \mathfrak{m}$ be an ideal and $n>0$ an integer. Then the following are equivalent:

(1) $\ell\left(\operatorname{Ext}_{R}^{i}(R / I, M)\right)<\infty$, for all $i<n$;

(2) I contains an $M$-filter regular sequence of length $n$.

When $x_{1}, \ldots, x_{n} \in I$ is an $M$-filter regular sequence,

$$
\operatorname{Ext}_{R}^{n}(R / I, M)_{\mathfrak{p}} \cong \operatorname{Hom}_{R}\left(R / I, M /\left(x_{1}, \ldots, x_{n}\right) M\right)_{\mathfrak{p}}, \text { for any } \mathfrak{p} \in \operatorname{Spec}(R) \backslash\{\mathfrak{m}\} \text {. }
$$

Proof. Assume that $\ell\left(\operatorname{Ext}_{R}^{i}(R / I, M)\right)<\infty$, for all $i<n$. We use induction on $n$ to show that $I$ contains an $M$-filter regular sequence of length $n$. If $n=1$, then $I$ contains an $M$-filter regular element by Lemma 3.1. Now assume that $n>1$ and the result is true for $n-1$. Then, by Lemma 3.1 again, there is $x_{1} \in I$ which is $M$-filter regular. From the short exact sequences

$$
\begin{aligned}
& 0 \rightarrow 0: M x_{1} \rightarrow M \stackrel{x_{1}}{\rightarrow} x_{1} M \rightarrow 0, \\
& 0 \rightarrow x_{1} M \rightarrow M \rightarrow M / x_{1} M \rightarrow 0,
\end{aligned}
$$

we get the long exact sequences

$$
\begin{gathered}
0 \rightarrow \operatorname{Hom}_{R}\left(R / I, 0:_{M} x_{1}\right) \rightarrow \operatorname{Hom}_{R}(R / I, M) \stackrel{x_{1}}{\rightarrow} \operatorname{Hom}_{R}\left(R / I, x_{1} M\right) \\
\rightarrow \operatorname{Ext}_{R}^{1}\left(R / I, 0:_{M} x_{1}\right) \rightarrow \cdots, \\
0 \rightarrow \operatorname{Hom}_{R}\left(R / I, x_{1} M\right) \rightarrow \operatorname{Hom}_{R}(R / I, M) \rightarrow \operatorname{Hom}_{R}\left(R / I, M / x_{1} M\right) \\
\rightarrow \operatorname{Ext}_{R}^{1}\left(R / I, x_{1} M\right) \rightarrow \cdots .
\end{gathered}
$$

As $\ell\left(0:_{M} x_{1}\right)<\infty$, we have that $\ell\left(\operatorname{Ext}_{R}^{i}\left(R / I, 0:_{M} x_{1}\right)\right)<\infty$ for any $i \geq 0$. Then we see that $\ell\left(\operatorname{Ext}_{R}^{i}\left(R / I, x_{1} M\right)\right)<\infty$ for all $i<n$ from the first long exact sequence. From the second long exact sequence, we get that $\ell\left(\operatorname{Ext}_{R}^{i}\left(R / I, M / x_{1} M\right)\right)<\infty$ for all $i<n-1$. Then, by the induction assumption, there exist $x_{2}, \ldots, x_{n} \in I$ which 
is an $M / x_{1} M$-filter regular sequence. Hence $x_{1}, x_{2}, \ldots, x_{n}$ is an $M$-filter regular sequence.

Conversely, suppose that $I$ contains an $M$-filter regular sequence of length $n$. Let $x_{1}, \ldots, x_{n} \in I$ be an $M$-filter regular sequence. For any $\mathfrak{p} \in \operatorname{Supp}(M / I M) \backslash\{\mathfrak{m}\}$, $x_{1} / 1, \ldots, x_{n} / 1$ is an $M_{\mathfrak{p}}$-regular sequence. Then, by a well-known property of $M_{\mathfrak{p}}$-regular sequences, we have

$$
\operatorname{Ext}_{R_{\mathfrak{p}}}^{i}\left(R_{\mathfrak{p}} / I R_{\mathfrak{p}}, M_{\mathfrak{p}}\right)=0, \text { for all } i<n,
$$

and

$$
\operatorname{Ext}_{R_{\mathfrak{p}}}^{n}\left(R_{\mathfrak{p}} / I R_{\mathfrak{p}}, M_{\mathfrak{p}}\right) \cong \operatorname{Hom}_{R_{\mathfrak{p}}}\left(R_{\mathfrak{p}} / I R_{\mathfrak{p}}, M_{\mathfrak{p}} /\left(x_{1} / 1, \ldots, x_{n} / 1\right) M_{\mathfrak{p}}\right) .
$$

Thus

$$
\begin{gathered}
\operatorname{Ext}_{R}^{i}(R / I, M)_{\mathfrak{p}}=0, \text { for all } i<n, \\
\operatorname{Ext}_{R}^{n}(R / I, M)_{\mathfrak{p}} \cong \operatorname{Hom}_{R}\left(R / I, M /\left(x_{1}, \ldots, x_{n}\right) M\right)_{\mathfrak{p}} .
\end{gathered}
$$

But, for any $\mathfrak{p} \notin \operatorname{Supp}(M / I M)$ and any $i \geq 0$, it is clear that

$$
\operatorname{Ext}_{R}^{i}(R / I, M)_{\mathfrak{p}}=0 \text { and } \operatorname{Hom}_{R}\left(R / I, M /\left(x_{1}, \ldots, x_{n}\right) M\right)_{\mathfrak{p}}=0 .
$$

Hence $\ell\left(\operatorname{Ext}_{R}^{i}(R / I, M)\right)<\infty$ for all $i<n$ and for any $\mathfrak{p} \in \operatorname{Spec}(R) \backslash\{\mathfrak{m}\}$,

$$
\operatorname{Ext}_{R}^{n}(R / I, M)_{\mathfrak{p}} \cong \operatorname{Hom}_{R}\left(R / I, M /\left(x_{1}, \ldots, x_{n}\right) M\right)_{\mathfrak{p}} .
$$

This completes the proof.

If $x_{1}, \ldots, x_{n}$ is a maximal $M$-filter regular sequence in $I$, then, by Lemma 3.1, $\operatorname{dim}\left(\operatorname{Hom}_{R}\left(R / I, M /\left(x_{1}, \ldots, x_{n}\right) M\right)\right)>0$. It follows from Proposition 3.2 that $\operatorname{dim}\left(\operatorname{Ext}_{R}^{n}(R / I, M)\right)>0$. Hence any two maximal $M$-filter regular sequences in $I$ (if any exist) have the same length.

Definition 3.3. Let $I$ be a proper ideal of $R$. The f-depth (filter depth) of $I$ on $M$ is defined as the length of any maximal $M$-filter regular sequence in $I$, denoted by f-depth $(I, M)$. Here, when the maximal $M$-filter regular sequence in $I$ does not exist, we understand that the length is $\infty$.

Notice that $\mathrm{f}-\operatorname{depth}(I, M)=0$ if and only if $I \subseteq \mathfrak{p}$ for some $\mathfrak{p} \in \operatorname{Ass}_{R}(M) \backslash\{\mathfrak{m}\}$ and if $x \in I$ is $M$-filter regular, then

$$
\mathrm{f}-\operatorname{depth}(I, M)=\mathrm{f}-\operatorname{depth}(I, M / x M)+1 .
$$

Furthermore, by Proposition 3.2, we have that

$$
\text { f-depth }(I, M)=\min \left\{n \mid \operatorname{dim}\left(\operatorname{Ext}_{R}^{n}(R / I, M)\right)>0\right\},
$$

where, when $\operatorname{dim}\left(\operatorname{Ext}_{R}^{n}(R / I, M)\right) \leq 0$ for all $n \geq 0$, we understand the right side of the above equality to be $\infty$.

Proposition 3.4. Let $I, J$ be proper ideals of $R$. If $\sqrt{I}=\sqrt{J}$, then

$$
f \text {-depth }(I, M)=f \text {-depth }(J, M) .
$$

Proof. Let $x_{1}, \ldots, x_{n} \in I$ be an $M$-filter regular sequence. Then, as $\sqrt{I}=\sqrt{J}$, there exists an integer $\alpha>0$ such that $x_{1}^{\alpha}, \ldots, x_{n}^{\alpha} \in J$. But since $x_{1}^{\alpha}, \ldots, x_{n}^{\alpha}$ is also $M$-filter regular, we see that

$$
\mathrm{f}-\operatorname{depth}(I, M) \leq \mathrm{f}-\operatorname{depth}(J, M) .
$$

Similarly,

$$
\mathrm{f}-\operatorname{depth}(J, M) \leq \mathrm{f}-\operatorname{depth}(I, M) .
$$

Thus f-depth $(I, M)=\mathrm{f}-\operatorname{depth}(J, M)$. 
Suppose that $\operatorname{dim}(M / I M)>0$. If $x \in I$ is $M$-filter regular, then $x \notin \mathfrak{p}$, for any $\mathfrak{p} \in \operatorname{Ass}_{R}(M)$ with $\operatorname{dim}(R / \mathfrak{p})=\operatorname{dim}(M)$. Thus $\operatorname{dim}(M / x M)=\operatorname{dim}(M)-1$. It follows that every $M$-filter regular sequence in $I$ is a subsystem of parameters for $M$. Furthermore, we have the following:

Proposition 3.5. If $\operatorname{dim}(M / I M)>0$, then

$$
\operatorname{depth}(I, M) \leq f-\operatorname{depth}(I, M) \leq h t_{M} I,
$$

where $h t_{M} I$ is the infimum of lengths of strictly decreasing chains of prime ideals in $\operatorname{Supp}(M)$ starting from a prime ideal containing $I$.

Proof. As any $M$-regular sequence is an $M$-filter regular sequence, we see that $\operatorname{depth}(I, M) \leq \mathrm{f}-\operatorname{depth}(I, M)$. So it remains to $\operatorname{show}$ that $\mathrm{f}-\operatorname{depth}(I, M) \leq \mathrm{ht}_{M}(I)$.

By assumption, $\operatorname{Supp}(M / I M) \nsubseteq\{\mathfrak{m}\}$. Let $x_{1}, \ldots, x_{n} \in I$ be any $M$-filter regular sequence. It is enough to show that $n \leq \mathrm{ht}_{M}(\mathfrak{p})$ for any $\mathfrak{p} \in \operatorname{Supp}(M / I M) \backslash\{\mathfrak{m}\}$. As $x_{1}, \ldots, x_{n} \in \mathfrak{p}, x_{1} / 1, \ldots, x_{n} / 1 \in R_{\mathfrak{p}}$ is an $M_{\mathfrak{p}}$-regular sequence. Hence $n \leq$ $\operatorname{dim}\left(M_{\mathfrak{p}}\right)=\mathrm{ht}_{M}(\mathfrak{p})$, as required.

Proposition 3.6. $f$-depth $(I, M)=\infty$ if and only if I contains a system of parameters for $M$.

Proof. Notice that $I$ containing a system of parameters for $M$ is equivalent to $\operatorname{dim}(M / I M)=0$. Thus, by Proposition 3.5, we only need to show that, when $\operatorname{dim}(M / I M)=0$, for any integer $n>0$ we can find an $M$-filter regular sequence of length $n$ in $I$. But, in this case, we have that $\ell\left(\operatorname{Ext}_{R}^{i}(R / I, M)\right)<\infty$ for all $i \geq 0$. Then the result follows from Proposition 3.2.

Proposition 3.7. Let $V(I)$ be the set of prime ideals containing I. Then

$$
f \text {-depth }(I, M)=\min \{f-\operatorname{depth}(\mathfrak{p}, M) \mid \mathfrak{p} \in V(I)\} .
$$

Proof. If $\operatorname{dim}(M / I M)=0$, then $\mathrm{f}-\operatorname{depth}(I, M)=\infty$. But since the prime ideal containing $I$ is just $\mathfrak{m}$ and $\mathfrak{f}-\operatorname{depth}(\mathfrak{m}, M)=\infty$, the equality holds. Now assume that $\operatorname{dim}(M / I M)>0$. Set $r=\min \{f-\operatorname{depth}(\mathfrak{p}, M) \mid \mathfrak{p} \in V(I)\}$. As there is some $\mathfrak{p} \in V(I)$ such that $\operatorname{dim}(M / \mathfrak{p} M)>0$, we have that $\mathrm{f}-\operatorname{depth}(\mathfrak{p}, M)<\infty$ hence, $r<\infty$. We use induction on $r$ to show that $\mathrm{f}-\operatorname{depth}(I, M)=r$. If $r=0$, then there exists a prime ideal $\mathfrak{p} \supseteq I$ such that $\mathrm{f}$ - $\operatorname{depth}(\mathfrak{p}, M)=0$. Thus, as $I \subseteq \mathfrak{p}$, $\mathrm{f}$ - $\operatorname{depth}(I, M)=0$. Suppose that $r>0$. Then, for any $\mathfrak{p} \in \operatorname{Ass}_{R}(M) \backslash\{\mathfrak{m}\}$, as $\mathrm{f}$ - $\operatorname{depth}(\mathfrak{p}, M)=0$ and $r>0$, we have $I \nsubseteq \mathfrak{p}$. Hence $I \nsubseteq \mathbb{U} \mathfrak{p}$. Thus there exists $x_{1} \in I$ which is $M$-filter regular. Set $M_{1}=M / \mathfrak{p} \in \operatorname{xss}_{1} M$. Then

$$
\min \left\{\mathrm{f}-\operatorname{depth}\left(\mathfrak{p}, M_{1}\right) \mid \mathfrak{p} \in V(I)\right\}=\min \{\mathrm{f}-\operatorname{depth}(\mathfrak{p}, M)-1 \mid \mathfrak{p} \in V(I)\}=r-1 .
$$

Hence, by the induction assumption, $\mathrm{f}-\operatorname{depth}\left(I, M_{1}\right)=r-1$, so f-depth $(I, M)=$ $\mathrm{f}-\operatorname{depth}\left(I, M_{1}\right)+1=r$, as required.

For any finitely generated $R$-module $N$, its m-adic completion is denoted by $\widehat{N}$. The following proposition states that f-depth does not change after passing to completion.

Proposition 3.8. $f$-depth $(I, M)=f-\operatorname{depth}(\widehat{I}, \widehat{M})$. 
Proof. This is because, for any $i \geq 0$,

$$
\left.\operatorname{dim}\left(\operatorname{Ext}_{R}^{i}(R / I, M)\right)=\operatorname{dim}\left(\operatorname{Ext}_{R}^{i} \widehat{(R / I}, M\right)\right)=\operatorname{dim}\left(\operatorname{Ext}_{\widehat{R}}^{i}(\widehat{R} / \widehat{I}, \widehat{M})\right) .
$$

The following theorems give two characterizations of f-depth.

Theorem 3.9. Let $y_{1}, \ldots, y_{n} \in I$ such that $I=\left(y_{1}, \ldots, y_{n}\right)$. Then

$$
f-\operatorname{depth}(I, M)=n-\sup \left\{i \mid \operatorname{dim}\left(H_{i}\left(y_{1}, \ldots, y_{n} ; M\right)\right)>0\right\},
$$

where, if there is no integer $i$ with $\operatorname{dimH}_{i}\left(y_{1}, \ldots, y_{n} ; M\right)>0$, we understand that the right side of the above equality is $\infty$.

Proof. If $\operatorname{dim}(M / I M)=0$, then $\mathrm{f}-\operatorname{depth}(I, M)=\infty$ and $\operatorname{dim}\left(H_{i}\left(y_{1}, \ldots, y_{n} ; M\right)\right) \leq$ 0 for any $i$ (since $I \cdot H_{i}\left(y_{1}, \ldots, y_{n} ; M\right)=0$ ), so the theorem is true in this case. Now assume that $\operatorname{dim}(M / I M)>0$. Let $r=\mathrm{f}-\operatorname{depth}(I, M)$. We use induction on $r$. If $r=0$, then $I \subseteq \mathfrak{p}$ for some $\mathfrak{p} \in \operatorname{Ass}_{R}(M) \backslash\{\mathfrak{m}\}$. Thus $\mathfrak{p}=\operatorname{ann}_{R}(m)$ for some $m \in M$. As $I m=0$, we see that $m \in 0: M I=H_{n}\left(y_{1}, \ldots, y_{n} ; M\right)$. Then $\mathfrak{p} \in$ $\operatorname{Ass}_{R}\left(H_{n}\left(y_{1}, \ldots, y_{n} ; M\right)\right)$. But since $\mathfrak{p} \neq \mathfrak{m}$, we have that $\operatorname{dim}\left(H_{n}\left(y_{1}, \ldots, y_{n} ; M\right)\right)>$ 0 , and the equality holds. Suppose that $r>0$. Let $x \in I$ be an $M$-filter regular element and $M_{1}=M / x M$. Then, as $\mathrm{f}-\operatorname{depth}\left(I, M_{1}\right)=r-1$, we have, by the induction assumption, that

$$
\sup \left\{i \mid \operatorname{dim}\left(H_{i}\left(y_{1}, \ldots, y_{n} ; M_{1}\right)>0\right\}=n-r+1 .\right.
$$

Note that, as $\operatorname{Supp}\left(H_{i}\left(y_{1}, \ldots, y_{n} ; M_{1}\right)\right) \subseteq \operatorname{Supp}(M / I M)$, the above equality is equivalent to $H_{i}\left(y_{1}, \ldots, y_{n} ; M_{1}\right)_{\mathfrak{p}}=0$ for all $i>n-r+1$ and any $\mathfrak{p} \in \operatorname{Supp}(M / I M) \backslash$ $\{\mathfrak{m}\}$, and $H_{n-r+1}\left(y_{1}, \ldots, y_{n} ; M_{1}\right)_{\mathfrak{p}} \neq 0$ for some $\mathfrak{p} \in \operatorname{Supp}(M / I M) \backslash\{\mathfrak{m}\}$.

For any $\mathfrak{p} \in \operatorname{Supp}(M / I M) \backslash\{\mathfrak{m}\}$, as $x \in \mathfrak{p}$, we see that $x / 1$ is $M_{\mathfrak{p}}$-regular. From the short exact sequence

$$
0 \rightarrow M_{\mathfrak{p}} \stackrel{x / 1}{\rightarrow} M_{\mathfrak{p}} \rightarrow\left(M_{1}\right)_{\mathfrak{p}} \rightarrow 0
$$

we have a long exact sequence

$$
\begin{gathered}
\cdots \rightarrow H_{i}\left(y_{1} / 1, \ldots, y_{n} / 1 ; M_{\mathfrak{p}}\right) \stackrel{x / 1}{\rightarrow} H_{i}\left(y_{1} / 1, \ldots, y_{n} / 1 ; M_{\mathfrak{p}}\right) \\
\rightarrow H_{i}\left(y_{1} / 1, \ldots, y_{n} / 1 ;\left(M_{1}\right)_{\mathfrak{p}}\right) \rightarrow H_{i-1}\left(y_{1} / 1, \ldots, y_{n} / 1 ; M_{\mathfrak{p}}\right) \stackrel{x / 1}{\rightarrow} \cdots .
\end{gathered}
$$

As $H_{i}\left(y_{1} / 1, \ldots, y_{n} / 1 ; M_{\mathfrak{p}}\right)$ is annihilated by $x / 1$, the above long exact sequence is split into short exact sequences

$$
\begin{gathered}
0 \rightarrow H_{i}\left(y_{1} / 1, \ldots, y_{n} / 1 ; M_{\mathfrak{p}}\right) \rightarrow H_{i}\left(y_{1} / 1, \ldots, y_{n} / 1 ;\left(M_{1}\right)_{\mathfrak{p}}\right) \\
\rightarrow H_{i-1}\left(y_{1} / 1, \ldots, y_{n} / 1 ; M_{\mathfrak{p}}\right) \rightarrow 0
\end{gathered}
$$

i.e.,

$$
0 \rightarrow H_{i}\left(y_{1}, \ldots, y_{n} ; M\right)_{\mathfrak{p}} \rightarrow H_{i}\left(y_{1}, \ldots, y_{n} ; M_{1}\right)_{\mathfrak{p}} \rightarrow H_{i-1}\left(y_{1}, \ldots, y_{n} ; M\right)_{\mathfrak{p}} \rightarrow 0 .
$$

Then $H_{i}\left(y_{1}, \ldots, y_{n} ; M\right)_{\mathfrak{p}}=0$ for any $i>n-r$ and any $\mathfrak{p} \in \operatorname{Supp}(M / I M) \backslash$ $\{\mathfrak{m}\}$, and $H_{n-r}\left(y_{1}, \ldots, y_{n} ; M\right)_{\mathfrak{p}} \neq 0$ for some $\mathfrak{p} \in \operatorname{Supp}(M / I M) \backslash\{\mathfrak{m}\}$. Hence $\sup \left\{i \mid \operatorname{dim}\left(H_{i}\left(y_{1}, \ldots, y_{n} ; M\right)\right)>0\right\}=n-r$. The theorem follows.

Theorem 3.10 (4, Theorem 3.1]). For any proper ideal I of R,

$$
f \text {-depth }(I, M)=\min \left\{r \mid H_{I}^{r}(M) \text { is not Artinian }\right\} .
$$


Proof. If $\operatorname{dim}(M / I M)=0$, then $\sqrt{I+\operatorname{ann}_{R}(M)}=\mathfrak{m}$, hence, $H_{I}^{r}(M) \cong H_{\mathfrak{m}}^{r}(M)$ is Artinian for any $r \geq 0$. Thus $\min \left\{r \mid H_{I}^{r}(M)\right.$ is not Artinian $\}=\infty$. In this case, $\mathrm{f}-\operatorname{depth}(I, M)=\infty$ and the result is true.

Now we assume that $\operatorname{dim}(M / I M)>0$. Let $n=\mathrm{f}-\operatorname{depth}(I, M)$. Then $n=$ $\min \left\{i \mid \operatorname{dim}\left(\operatorname{Ext}_{R}^{i}(R / I, M)\right)>0\right\}$. Note that $\operatorname{dim}\left(\operatorname{Ext}_{R}^{i}(R / I, M)\right) \leq 0$ for all $i<n$ and $\operatorname{dim}\left(\operatorname{Ext}_{R}^{n}(R / I, M)\right)>0$ is equivalent to $\operatorname{Ext}_{R_{\mathfrak{p}}}^{i}\left(R_{\mathfrak{p}} / I R_{\mathfrak{p}}, M_{\mathfrak{p}}\right)=0$ for any $i<n$ and any $\mathfrak{p} \in \operatorname{Supp}(M / I M) \backslash\{\mathfrak{m}\}$, but, $\operatorname{Ext}_{R_{\mathfrak{p}}}^{n}\left(R_{\mathfrak{p}} / I R_{\mathfrak{p}}, M_{\mathfrak{p}}\right) \neq 0$ for some $\mathfrak{p} \in \operatorname{Supp}(M / I M) \backslash\{\mathfrak{m}\}$; i.e., $\operatorname{depth}\left(I R_{\mathfrak{p}}, M_{\mathfrak{p}}\right) \geq n$ for any $\mathfrak{p} \in \operatorname{Supp}(M / I M) \backslash\{\mathfrak{m}\}$ and $\operatorname{depth}\left(I R_{\mathfrak{p}}, M_{\mathfrak{p}}\right)=n$ for some $\mathfrak{p} \in \operatorname{Supp}(M / I M) \backslash\{\mathfrak{m}\}$. It follows that

$$
n=\min \left\{\operatorname{depth}\left(I R_{\mathfrak{p}}, M_{\mathfrak{p}}\right) \mid \mathfrak{p} \in \operatorname{Supp}(M / I M) \backslash\{\mathfrak{m}\}\right\} .
$$

Then, by [4, Theorem 3.1], $n=\min \left\{r \mid H_{I}^{r}(M)\right.$ is not Artinian $\}$.

\section{F-DEPTH AND F-MODUles}

A finitely generated $R$-module $M$ is called an f-module if every system of parameters for $M$ is an $M$-filter regular sequence. F-modules were introduced in [1] as a generalization of Cohen-Macaulay modules.

The following theorem gives a characterization of f-modules by f-depth.

Theorem 4.1. $M$ is an f-module if and only if, for any $\mathfrak{p} \in \operatorname{Supp}(M) \backslash\{\mathfrak{m}\}$,

$$
f \text {-depth }(\mathfrak{p}, M)=\operatorname{dim}(M)-\operatorname{dim}(R / \mathfrak{p}) .
$$

Proof. Suppose that $M$ is an f-module. Let $\mathfrak{p} \in \operatorname{Supp}(M) \backslash\{\mathfrak{m}\}$ and $x_{1}, \ldots, x_{r}$ be a maximal $M$-filter regular sequence in $\mathfrak{p}$. Then

$$
\mathfrak{p} \subseteq \underset{\mathfrak{q} \in \operatorname{Ass}_{R}\left(M /\left(x_{1}, \ldots, x_{r}\right) M\right) \backslash\{\mathfrak{m}\}}{\bigcup} \mathfrak{q}
$$

hence, $\mathfrak{p} \subseteq \mathfrak{q}$ for some $\mathfrak{q} \in \operatorname{Ass}_{R}\left(M /\left(x_{1}, \ldots, x_{r}\right) M\right) \backslash\{\mathfrak{m}\}$. As $M$ is an f-module and $x_{1}, \ldots, x_{r}$ is a subsystem of parameters for $M$, we have, by [1, (2.5)], that $\operatorname{dim}(R / \mathfrak{q})=\operatorname{dim}\left(M /\left(x_{1}, \ldots, x_{r}\right) M\right)$. But since $\mathfrak{p} \in \operatorname{Supp}\left(M /\left(x_{1}, \ldots, x_{r}\right) M\right)$, we see that $\mathfrak{p}=\mathfrak{q}$, hence

$$
\mathrm{f}-\operatorname{depth}(\mathfrak{p}, M)=r=\operatorname{dim}(M)-\operatorname{dim}(R / \mathfrak{p}) .
$$

Conversely, suppose that

$$
\mathrm{f}-\operatorname{depth}(\mathfrak{p}, M)=\operatorname{dim}(M)-\operatorname{dim}(R / \mathfrak{p})
$$

for all $\mathfrak{p} \in \operatorname{Supp}(M) \backslash\{\mathfrak{m}\}$. We use induction on $d:=\operatorname{dim}(M)$ to show that $M$ is an f-module. The case $d=0$ is trivial. Suppose that $d>0$. Let $x_{1}, x_{2}, \ldots, x_{d}$ be a system of parameters for $M$. Then $x_{1}$ is $M$-filter regular, otherwise $x_{1} \in \mathfrak{p}$ for some $\mathfrak{p} \in \operatorname{Ass}_{R}(M) \backslash\{\mathfrak{m}\}$, hence

$$
\text { f-depth }(\mathfrak{p}, M)+\operatorname{dim}(R / \mathfrak{p})=\operatorname{dim}(R / \mathfrak{p}) \leq \operatorname{dim}\left(M / x_{1} M\right)=\operatorname{dim}(M)-1,
$$

a contradiction. Set $M_{1}=M / x_{1} M$. Then $\operatorname{dim}\left(M_{1}\right)=d-1$ and $x_{2}, \ldots, x_{d}$ is a system of parameters for $M_{1}$ and, for any $\mathfrak{p} \in \operatorname{Supp}\left(M_{1}\right) \backslash\{\mathfrak{m}\}$, as $x_{1} \in \mathfrak{p}$, we have that

$$
\begin{aligned}
\mathrm{f}-\operatorname{depth}\left(\mathfrak{p}, M_{1}\right) & =\mathrm{f}-\operatorname{depth}(\mathfrak{p}, M)-1=\operatorname{dim}(M)-\operatorname{dim}(R / \mathfrak{p})-1 \\
& =\operatorname{dim}\left(M_{1}\right)-\operatorname{dim}(R / \mathfrak{p}) .
\end{aligned}
$$

Thus, by induction assumption, $x_{2}, \ldots, x_{d}$ is an $M_{1}$-filter regular sequence. Then $x_{1}, x_{2}, \ldots, x_{d}$ is an $M$-filter regular sequence and $M$ is an f-module. 
Remark 4.2. Suppose that $M$ is an f-module. Let $I$ be a proper ideal of $R$ such that $I \supseteq \operatorname{ann}_{R}(M)$ and $\sqrt{I} \neq \mathfrak{m}$. Then, by Proposition 3.7 and Theorem 4.1, we have that

$$
\begin{aligned}
\mathrm{f}-\operatorname{depth}(I, M) & =\min \{\mathrm{f}-\operatorname{depth}(\mathfrak{p}, M) \mid \mathfrak{p} \in \operatorname{Supp}(M / I M) \backslash\{\mathfrak{m}\}\} \\
& =\operatorname{dim}(M)-\max \{\operatorname{dim}(R / \mathfrak{p}) \mid \mathfrak{p} \in \operatorname{Supp}(M / I M) \backslash\{\mathfrak{m}\}\} \\
& =\operatorname{dim}(M)-\operatorname{dim}(R / I) .
\end{aligned}
$$

Note that $\operatorname{ht}_{M}(I) \leq \operatorname{dim}(M)-\operatorname{dim}(R / I)$ and it follows from Proposition 3.5 that

$$
\mathrm{f}-\operatorname{depth}(I, M)=\operatorname{ht}_{M}(I)=\operatorname{dim}(M)-\operatorname{dim}(R / I) .
$$

\section{REFERENCES}

[1] Cuong, N.T., P. Schenzel, N. V. Trung: Verallgemeinerte Cohen-Macaulay-Moduln, Math. Nachr. 85(1978),57-73. MR 80i:13008

[2] Faltings, G.: Über die Annulatoren lokaler Kohomologiegruppen, Arch. Math. 30(1978), 473476. MR 58:22058

[3] Matsumura, H.: Commutative Ring Theory. Cambridge Uni. Press, 1986. MR 88h:13001

[4] Melkersson, L.: Some applications of a criterion for artinianness of a module, J. Pure and Appl. Math. 01(1995),291-303. MR 96b:13044

[5] Sharp, R. Y.: Local cohomology theory in commutative algebra, Quart. J. Math. (Oxford), 21(1970), 425-434. MR 43:1965

Department of mathematics, Suzhou University, Suzhou 215006, People's Republic of CHINA

Department of mathematics, Suzhou University, Suzhou 215006, People's Republic of CHINA

E-mail address: zmtang@suda.edu.cn 